\title{
PETRUS VAN MUSSCHENBROEK Y LA FÍSICA EXPERIMENTAL DEL SIGLO XVIII
}

\author{
Víctor Guijarro Mora
}

Universidad Rey Juan Carlos

\section{RESUMEN}

El artículo se centra en el estudio del significado y alcance de la física experimental en el siglo XVIII. En particular, se analiza el papel preciso que desempeñaron los diferentes tipos de experimentos planteados dentro de esta concepción de los fenómenos naturales así como la importancia que el rechazo de las matemáticas (en especial la geometría) tuvo entre los físicos experimentales en sentido estricto.

PALABRAS CLAVE: física experimental, siglo XVIII, Petrus van Musschembroek.

\section{SUMMARY}

The paper deals with the meaning and scope of Eigtheenth-century experimental physics. In particular, it is focused on anlysing the price role played by the different type of experiments considered among the strict experimental physicists. On the other hand, the paper is intended to account for one of the basic principles of these natural philosophers: the rejection of mathematics, especially the geometrical approach to nature.

KEY WORDS: experimental physics, $18^{\text {th }}$ century, Petrus van Musschembroek.

\section{INTRODUCCIÓN}

A lo largo de la Ilustración, la física conoció una transformación fundamental en los métodos y en los contenidos. Estimulada por las novedades y resultados introducidos en el siglo anterior por Descartes, Galileo, Boyle y Newton, entre otros, durante este período se consolidó el distanciamiento entre este nuevo modelo de filosofía natural y el tradicional inspirado en Aristóteles, dominante aún en muchos centros de enseñanza superior. En este proceso de cambio fue fundamental la aportación y desarrollo de dos orientaciones que conformaron la física ilustrada: la mecánica práctica y la física experimental. Las líneas que siguen estarán centradas en determinar en qué consistió esta última vertiente de las ciencias físicas y cuál fue su contribución a la reforma mencionada. 


\section{VÍCTOR GUIJARRO MORA}

Básicamente la mecánica práctica estaba dedicada a estudiar las condiciones de aplicación de los principios mecánicos generales a la descripción de situaciones concretas. Así, comprendía, por ejemplo, diferentes ramas de la hidrostática y otras tantas de la mecánica, como las relacionadas con la resistencia de materiales o con el análisis del rozamiento. Por su parte, la física experimental, que no tenía la tradición de la anterior ni contenía principios generales comparables, esencialmente participó de los rasgos comunes a la física en general ${ }^{1}$ y que, a su vez, constituyeron los elementos sobre los que se cimentó el cambio de las concepciones tradicionales. En primer lugar, el alcance de sus contenidos era más limitado que en el enfoque antiguo: no comprendía ni las investigaciones relacionadas con el mundo orgánico ni las referidas al hombre. En segundo lugar, se justificaba el uso de las matemáticas como modo de representación de las regularidades de la naturaleza. En tercer lugar, partían de una teoría de la materia basada en alguna de las versiones de la filosofía mecánico-corpuscular. Y, por último, la experimentación recibió un importante impulso como vía de indagación de la naturaleza y como recurso demostrativo de las teorías. Sin embargo, algunas peculiaridades, como el papel preciso reservado a las matemáticas así como el uso y alcance de la experimentación, convirtieron a esta disciplina en un saber científico diferenciable tanto de la mecánica como de otro enfoque, desarrollado en el seno de la física, que contó con contribuciones a lo largo de todo el siglo: la física experimental matemática².

Así pues, durante el Siglo de las Luces, se desarrollaron tres orientaciones que convivieron durante este período, relacionadas unas con las llamadas matemáticas mixtas y otras con la filosofía natural. Las tres aprovecharán las ventajas del experimento, y por ello en la historiografía se ha asociado con la disciplina conocida como Física experimental; sin embargo dado que cada una perseguía finalidades diferentes y contaban también con planteamientos distintos, en este trabajo se va a establecer una distinción que va a corresponder con las siguientes opciones:

1 Sobre la física de este siglo, CoHEN, I. B. (1956), Franklin and Newton. An Inquiry into Speculative Newtonian Experimental Science and Franklin's Work in Electricity as an Example thereof, Philadelphia, The Philosophical Society; HeIlBron, J. L. (1970), Electricity in the 17th and 18th Centuries. A study of early modern physics, Berkeley, University of California Press; GUERLAC, H. (1981), trabajos reunidos en Newton on the Continent, Ithaca, Cornell University Press; GuERLAC, H. (1977), Essays and Papers in the History of Modern Science, Baltimore, The John Hopkins University Press y HANKINS, T. L. (1970), Jean D'Alembert. Science and the Enlightenment, Oxford, Clarendon Press.

2 Se prefiere esta acepción, que comprendería los trabajos, como se verá más adelante, de personajes con una sólida formación matemática, como F.U.T. Aepinus, J. H. Lambert o Ch. Coulomb, a la de «físicos matemáticos», ya que el uso de este concepto podría provocar alguna confusión con los estudios realizados en el ámbito de la mecánica (por ejemplo, los de J. L. D’Alembert o de L. Euler) o también con aproximaciones a la física más propias del siglo XIX, en concreto las procedentes de la escuela francesa inspirada en P. S. de Laplace (acerca de este último aspecto, FoX, R. (1974), «The Rise and Fall of Laplacian School», Historical Studies in the Physical Sciences, 4, 89-136). 
- En primer lugar, tenemos la mecánica práctica, incorporada en la física general, cuyo propósito era comprobar, partiendo de principios matemáticos, la aplicación de éstos a situaciones reales.

- En segundo lugar, la física experimental matemática, desarrollada a lo largo de todo el siglo, que empleó los experimentos en el sentido que lo hizo Newton en la Óptica, es decir, fundamentalmente para determinar propiedades generales que después pudieran ser matematizadas.

- Y, en tercer lugar, la física experimental en sentido estricto, de la que se hablará seguidamente, y que correspondía con la física particular y una parte de la física general.

El análisis que se llevará a cabo a continuación está destinado por tanto a dilucidar el sentido que adquirieron el uso de las matemáticas y el de la prueba experimental dentro de esta última rama de la física. Así se pondrá de manifiesto, primero, las particularidades de la física experimental (no matemática) frente a las otras opciones señaladas, y segundo, el alcance así como las limitaciones inherentes a este singular ámbito de estudios cuya difusión y desarrollo a lo largo de todo el siglo permitió la realización de importantes descubrimientos en la filosofía natural ${ }^{3}$. Atendiendo a estos propósitos, el trabajo se ha dividido en tres partes: la primera se ocupa de los contenidos de la física experimental, en la segunda se tratan los métodos de esta disciplina y en la tercera, por último, se muestra mediante diferentes ejemplos cómo estos presupuestos se pusieron en práctica.

Como punto de partida, he elegido la figura de Petrus van Musschenbroek, cuya obra, compuesta de numerosos manuales y otras tantas investigaciones en los campos del magnetismo, la electricidad y la calorimetría, representa de forma cabal el paradigma del físico experimental ilustrado tal y como se va a caracterizar en este artículo, esto es, el comprometido con la última de las orientaciones señaladas. Profesor de esta disciplina en la Universidad de Leiden (Holanda), sus esfuerzos se destinaron a recoger la herencia de los trabajos experimentales del siglo XVII (una de sus primeras publicaciones es una edición de los emblemáticos Saggi della Accademia del Cimento) y a sistematizar los logros y las abundantes cuestiones que aún permanecían abiertas en este ámbito. De aquí que nuestro personaje constituya un excelente punto de referencia para entender los aspectos asociados con estos estudios que trataremos a continuación.

3 Así pues, no se pretende en este limitado espacio dar cuenta completa del desarrollo de la física experimental en el siglo XVIII, pretensión que obligaría a atender a otras facetas de esta disciplina, como su proceso de institucionalización, los medios empleados en su difusión (manuales y salas de demostraciones públicas), sus aplicaciones prácticas (por ejemplo, en el ámbito de la medicina) y a sus elementos retóricos (estudiados, por ejemplo, por C. Licoppe en los capítulos 5º $6^{\circ}$ y $7^{\circ}$ de LICOPPE, C. (1996), $L a$ formation de la pratique scientifique. Le discours de l'experience en France et en Angleterre (16301820), París, La Découverte y en «Coulomb et la 'physique experimentale': pratique instrumentale et organisation narrative de la preuve», en BLONDEL, C. y DöRRIES, M., eds. (1994), Restaging Coulomb, Florencia, Leo S. Olschki, pp. 67-83). 


\section{VÍCTOR GUIJARRO MORA}

\section{EL PERFIL DE LA FÍSICA EXPERIMENTAL}

\section{Contenidos}

Para contar con una idea muy aproximada de los contenidos de la física es preciso, como aconseja J. L. Heilbron ${ }^{4}$, acudir a alguno de los numerosos manuales que se elaboraron durante este siglo. Entre todos ellos, los que mantienen un mínimo denominador común de las visiones de este período son los del mencionado Petrus van Musschenbroek. Varias razones, además de las ya apuntadas, justifican esta elección. En primer lugar, su producción reúne las innovaciones de la tradiciones continental e inglesa; segundo, su obra se enmarca entre 1730 y 1765, recogiendo las aportaciones de la primera mitad del siglo y anunciando las tendencias de la segunda. Otro elemento importante es la amplia difusión de sus escritos, que circularon por España, Portugal, Alemania, Inglaterra, Italia, Francia, y por supuesto, los Países Bajos. Y, por último, mantiene una posición característica de la época en relación con el uso de la experimentación y de las matemáticas en la física.

Musschenbroek estudió en la Universidad de Leiden, recibiendo el doctorado en medicina en 1715. Posteriormente realizó un viaje a Londres, donde conocería a Newton y el mundo científico de esta ciudad. A su vuelta comenzó a practicar la medicina, e influido por su hermano, cinco años mayor que él y fabricante de instrumentos, conoció a 's Gravesande, profesor en Leiden y autor de Physices, otro de los importantes manuales de física de ese siglo. En el año 1719 recibió el doctorado en filosofía, aceptando posteriormente el puesto de profesor de matemáticas y filosofía en la universidad de Duisburg (Alemania). Cuatro años más tarde abandonaba este cargo para trasladarse a la Universidad de Utrecht, donde se encargó del puesto de profesor de matemáticas, permaneciendo en este lugar hasta 1740, lugar en el que alcanzaría gran fama como físico experimentador. Finalmente entró en la Universidad de Leiden, ocupándose de la enseñanza de las matemáticas y de la filosofía y, a partir de la muerte de 's Gravesande, de la física experimental, puesto que ocupó hasta su fallecimiento en 1761.

La producción de Musschenbroek es extensa, destacando Epitome elementorum physico-mathematicorum conscripta in usus academicos (Utrecht, 1726), correspondiente a un resumen de sus lecciones; Essai de Physique (Leiden, 1739, $1^{\text {a }}$ ed.; 1751, $2^{\mathrm{a}}$ ), traducción francesa de un compendio más elaborado de las lecciones universitarias; Elementa physicae (Leiden, 1741), escrita expresamente para el público universitario (traducida al inglés en 1744 y al alemán en 1747), y Cours de physique expérimentale et mathematique (París, 1769), traducción al idioma galo de la Introductio

4 Heilbron, J. L., «Experimental Natural Philosophy», en RousseAu, G. S. \& PORTER, R. (1980), The Ferment of Knowledge. Studies in the Historiography of Eighteenth-Century Science, Cambridge, Cambridge University Press, p. 363. 
ad philosophiam naturalem (Lugduni Batavorum, 1762), el más importante de los trabajos del autor holandés 5 . De acuerdo con los manuales de Musschenbroek, la distribución de los temas que configuran el ámbito de desarrollo de la física experimental es la siguiente ${ }^{6}$ :

1. PROPIEDADES DE LOS CUERPOS. Consideraciones generales.

2. MECANICA. Cinemática, dinámica, percusión; máquinas, fricción; movimiento compuesto, péndulos, proyectiles; colisiones; resistencia de materiales.

3. MECANICA DE FLUIDOS. Tensión superficial, capilaridad; hidrostática, gravedad específica; higrometría; flujo de líquidos.

4. AGUA. Propiedades físicas; gravedad específica; congelación, ebullición, evaporación.

5. LUZ. Optica geométrica; colores, anillos de Newton; el ojo, instrumentos ópticos; luminiscencia.

6. AIRE. Gravedad específica, densidad; instrumentos pneumáticos; relaciones entre volumen y presión; sonido.

7. FUEGO. Pirómetros; espejos ustorios y lentes; termómetros; temperaturas de fusión; índices de absorción de calor; calorimetría; naturaleza de la llama, el calor y el fuego.

8. ELECTRICIDAD.

9. MAGNETISMO.

10. METEOROLOGIA. Formación de nubes, lluvia, granizo, nieve, rocío; arco iris, coronas, halos, parhelia; auroras, luz zodiacal, fuego lambente, relámpagos; vientos.

Los distintos saberes aquí enumerados, y algunos más que configuran la física, se distribuyen, según las concepciones generales de Musschenbroek, de acuerdo con las siguientes categorías:

Física experimental (cuerpos en general):

La parte general de la física experimental está basada en las propiedades generales de $1^{\mathrm{a}}$ y $2^{\mathrm{a}}$ clase $^{7}$, admitiendo la aplicación de las matemáticas. Las propiedades de primera clase comprenden, a su vez, la extensión, la impenetrabilidad, la fuerza de inercia, la movilidad, la reposabilidad y la figurabilidad, y las de segunda clase, la gravedad y la fuerza de atracción. En definitiva, todas las propiedades que afectan a la materia ordinaria.

5 Ruestow, E. G. (1973), Phyisics at 17th and 18th Century Leiden: Philosophy and the New Science in the University, La Haya, Martinus Nijhoff, p.120.

6 HEILBRON, «Experimental..», opus cit., pp. 366-7.

7 MusschenBroek, P. vAN (1751; 1ªed. 1736-39), Essai de physique, Leiden, pp. 22-23. 


\section{VÍCTOR GUIJARRO MORA}

Así, esta parte de la física comprende las siguientes ramas:

- Mecánica elemental

- Hidrostática elemental

- Hidrodinámica elemental

- Adherencia ${ }^{8}$

- Magnetismo ${ }^{9}$

La física particular, por su parte, está basada en las propiedades no comunes a todos los cuerpos. En este caso nos encontramos con una larga lista en la que aparecen referencias al calor, el sonido, la transparencia, la solidez, etc. Aquí, por tanto, se incluyen los siguientes estudios:

- Electricidad

- Agua

- Fuego

- Aire

- Luz

\section{Meteorología (fenómenos atmosféricos)}

- Meteoros aéreos

- Meteoros acuosos

- Meteoros ígneos

\section{Historia natural (cuerpos terrestres)}

Solamente la física experimental, como vemos, se ocupa de los cuerpos en general, lo cual la diferencia del resto de saberes, que se ocupan de aspectos particulares. Centrándonos ahora en esta disciplina, las propiedades generales que comprende admiten el aumento y la disminución, por lo que posibilitan la aplicación de las ma-

8 La adherencia se incluye en el primer grupo, así como también el magnetismo, porque dependen de la materia oridinaria; en los otros casos sería necesario un fluido especial. Sobre los trabajos experimentales realizados en el primero de estos ámbitos, que presuponen la existencia de fuerzas a corta distancia, MiLLINGTON, E. C. (1947), «Capillarity and Cohesion in the Eighteenth Century», Annals of Science, 5, 352-369.

9 En realidad, en este caso, Musschenbroek representa una excepción, ya que la tendencia dominante durante todo el siglo dentro de los físicos experimentales fue el apoyo con más o menos matices de la «teoría de la circulación», inspirada en Descartes; por tanto, estos estudios más bien deberían incluirse dentro de la física particular. Sobre este tema, HoME, R. W., «Magnetism», en AEPINUS, T. A. (1979), Essay on the Theory of Electricity and Magnetism, introd. y notas de R. W. Home, Princeton, Princeton University Press, pp. 137-188, espec. pp. 162-163. 
temáticas. En segundo lugar, la división entre la parte general y la particular de la física experimental no expresa una distancia infranqueable ${ }^{10}$ : una parte importante de las áreas contempladas en la física particular se encuentran conectadas con la general (por ejemplo, el estudio de las propiedades del agua o del aire derivadas de su peso). Además, al considerar la virtud atractiva como una propiedad general, cuyo estudio podía acometerse considerando sus efectos y sus leyes, algunos campos (electricidad y magnetismo) relegados a estudios descriptivos propios de las historias naturales podrían consolidarse como disciplinas características de la física general ${ }^{11}$. Por su parte, la meteorología, en cuanto que examina fenómenos vinculados con las propiedades del aire, del agua o de la electricidad puede remitirse a las conclusiones establecidas en la física particular o también en la general. Según este esquema, la fisica se distingue, por un lado, de la química en cuanto que a la segunda están reservadas aquellas propiedades particulares que no se remiten a ninguna de las generales ${ }^{12}, \mathrm{y}$ por el otro, de las matemáticas mixtas ${ }^{13}$ en que éstas solamente se ocupan de los cálculos prácticos derivados de las propiedades de primera clase (correspondiendo esto último con lo que antes se ha llamado «mecánica práctica»).

10 De hecho, en los textos de Musschenbroek no se reconoce explícitamente esta división dentro de la disciplina, solamente se establece en las propiedades (generales o particulares) que trata la física.

11 Esta fue la tendencia, como se verá más adelante (en la sección «Los métodos de la física experimental»), de los «físicos experimentales matemáticos», que en cambio los «físicos experimentales» no contemplarían.

12 G. F.Venel, en su artículo «Chymie» de la Encyclopédie (París, 1751, vol. 14), refuerza ese apreciación de distanciamiento entre la física y la química. Aquí apunta que la primera disciplina está destinada al estudio de las propiedades mecánicas de los cuerpos, fundadas en la masa, la presión, el peso, la fuerza, atendiendo además exclusivamente a la figura, el tamaño, el movimiento y la posición. Frente a estas cualidades «exteriores» se encuentran las «interiores o químicas», que son las que explican la afinidad, la separación de los corpúsculos por medio del calor y la volatilidad. Sobre la emancipación de la química, CRosland, M. (1980), «Chemistry and the Chemical Revolution», Rousseau, G. S. \& PORTER, R., opus cit., pp. 395-6.

13 En los siglos XVI, XVII y parte del XVIII se establecía una distinción entre matemáticas puras, que comprendían la geometría y la aritmética, y matemáticas mixtas, que incluían astronomía, óptica, estática, topografía, navegación, fortificación y artillería, entre otras. Aristóteles ya había destacado esta diferencia entre matemáticas puras y matemáticas «más próximas a la física», como la óptica, la armonía y la astronomía (ARISTÓTELES (1995), Física, 194a, 7-12; Madrid, Gredos, 1995, pp. 136-137); en la Metafísica incluye a la mecánica (ARISTÓTELES (1994),1078a 14-17; Madrid, Gredos, p. 513). Ahora bien, según el filósofo griego, aunque la matemática se interese, por ejemplo, por la figura de la Luna y el Sol, que son también competencia del físico, no las considera en tanto que límites de un cuerpo físico, «ni tampoco estudia los atributos mencionados en tanto que atributos de tales cuerpos. Por eso también los separan, pues por el pensamiento se los puede separar del movimiento» (Física, 193b, 33-35; opus cit., p. 136). 


\section{VÍCTOR GUIJARRO MORA}

\section{EL MARCO TEÓRICO DE LA FÍSICA: EL CORPUSCULARISMO}

Las concepciones de la materia existentes se dividían en función del uso de la hipótesis mecanicista y/o de la sustancialista (o materialista) ${ }^{14}$; la primera a su vez se dividía en dos, según se tuviera en cuenta la existencia de fuerzas o no. En el primer caso se trataba de corpúsculos impenetrables y dotados de inercia, que se movían en el vacío, y entre los que existían fuerzas a distancia; en el segundo se consideran partículas divisibles indefinidamente, dotadas de materia y movimiento, que actuaban entre ellas por impulso y presión dentro de un espacio material. Por otra parte, la sustancialista se apoyaba en la existencia de los llamados fluidos sutiles que, empleados para explicar, entre otros, los fenómenos eléctricos, magnéticos y térmicos, se concebían como sustancias con propiedades físicas, si bien diferenciables de la materia ordinaria (no añadían masa); por ejemplo, en este siglo se aceptó en general la noción de un fluido elástico calorífico, compuesto por partículas que se repelían entre sí y eran atraídas por la materia común.

Entre los representantes del mecanicismo del primer tipo se encuentran los trabajos en electricidad de J. A. Nollet. En cuanto al segundo, entre otros, cabe incluir a R. Cotes (1738) y J. Keill (1708), este último representante del corpuscularismo dinámico junto con J. T. Desaguliers (1734), R. Boscovitch (1758), J. Michell (1750) y H. Cavendish (1760). Por último, como sustancialistas, cabe mencionar a R. Green (1727), H. Boerhaave, H. Pemberton (1728), J. Priestley (1767) y J. Black (1770). Además existían posiciones intermedias, como la del mencionado Musschenbroek, que participaba de las diferentes tendencias (en calor y electricidad, sustancialista; en magnetismo y cohesión, mecanicista).

\section{LOS MÉTODOS DE LA FÍSICA EXPERIMENTAL}

Entre los promotores de estos estudios se prefirió la certeza de la experiencia a la evidencia de las matemáticas, por lo que la prueba experimental era considerada, como ya había sostenido R. Boyle en el siglo XVII, superior a la prueba matemáti$\mathrm{ca}^{15}$. Estas ideas se reforzaron con las aportaciones propias de la filosofía empirista

\footnotetext{
14 En general, sobre las diferentes posturas de la filosofía natural inglesa, SCHOFIELD, R. E. (1970), Mechanicism and Materialism. British Natural Philosophy in an Age of Reason, Princeton, Princeton University Press y las posteriores matizaciones a las rígidas dicotomías de este autor realizadas por P. M. Harman especialmente en (en colaboración con J. E. McGuire) «Newtonian Forces and Lockean Powers: Concepts of Matter in Eighteenth-Century Thought», Historical Studies in the Physical Sciences 3. Philadelphia, Pennsylvania, University of Pennsylvania Press, 1971, 233-306.

15 Sobre Boyle, Hall, M. B. (1965), Robert Boyle on natural philosophy, Bloomington, Indiana University Press, pp. 55-56.
} 
defendidas por J. Locke ${ }^{16}$. Para éste, si las ideas provenían de la experiencia, para comprobar la validez de cualquier constructo mental, era precisa su contrastación con la información sensible. Por estas razones, durante ese siglo y gran parte del siglo XVIII, una obra como los Principia Mathematica de Newton no fue considerada propiamente como un trabajo de física ya que partía de una entidad teórica, la fuerza, que era contemplada más bien como un producto de la imaginación ${ }^{17}$.

En el siglo XVIII, dos personajes, entre otros, apoyaron explícitamente estos presupuestos: J. T. Desaguliers y D. Diderot. El primero expresó su respaldo a estas posturas en la introducción del influyente Course of Experimental Philosophy (Londres, 1734-44) ${ }^{18}$, donde mantenía su confianza en la posibilidad de traducir a demostraciones experimentales los principios de filosofía natural de Newton. Por otra parte, el segundo, se refería a principios similares en su De la interpretación de la naturaleza (1753). Para Diderot, el cometido de la física no era «rectificar los cálculos de la geometría» ${ }^{19}$, sino constituirse en un conocimiento original de la naturaleza, el cual compartirían todos aquellos que practicasen el «arte experimental», es decir, los químicos, los físicos y los naturalistas. Las matemáticas, y el autor se fija principalmente en la geometría, son insuficientes para llevar a cabo este estudio porque su objeto es una abstracción exenta de cualidades individuales y sensibles; su finalidad es la aplicación de ciertas reglas de acuerdo con unas convenciones. Diderot, a partir de 1749, y el conde de Buffon, especialmente en su «Premier discourse de la manière d'étudier l'histoire naturelle», del mismo año, fueron los principales defensores de estas posturas en Francia. Junto a estos autores, defendieron este desinterés por las matemáticas G. Adams, B. Wilson, S. Hales, A. Volta, G. W. Richmann, B. Franklin, H. B. Saussure, J. A. Deluc y R.-J. Haüy ${ }^{20}$.

16 Sobre las relaciones entre esos dos autores, ALEXANDER, P. (1985), Ideas, qualities and corpuscles. Locke and Boyle on the external world, Cambridge, Cambridge University Press.

17 «...] la obra representaba un brillante despliegue de matemáticas, pero ignoraba los fundamentos físicos. En esto precisamente consistió la reacción de Christiaan Huygens y de la anónima recensión realizada para el Journal des sçavans. Tal y como era entendida la física, estos personajes tenían razón. Según especificaba el título de esta obra, Newton había establecido los principios matemáticos de la filosofía natural, no una filosofía natural según se concebía en ese momento», GuERLAC, H. (1977), «Where The Statue Stood: Divergent Loyalties to Newton in the Eighteenth Century», en Essays and Papers in the History of Modern Science, Baltimore, The John Hopkins Press, p. 140.

18 Sin entender la parte matemática de los Principia de Newton, Locke aceptó el newtonianismo, una anécdota que recoge Desaguliers en el prefacio de su obra: «Locke es el primero que se ha convertido al newtonianismo sin servirse de la geometría» (DESAGUliERS, J. T. (1751), Course de Physique Expérimentale, París, vii).

19 Diderot, D. (1992), De la interpretación de la naturaleza (ed. de Mariano Jalón y J. Mateo Ballorca), Barcelona, Anthropos, p. 7.

20 Dedicado principalmente a la mineralogía, realizando estudios de propiedades eléctricas de minerales así como de su composición geométrica, en 1802 escribió un tratado de física donde no incluía referencias a las aproximaciones matemáticas del siglo anterior, HoOYKAAS, R., «René-Just Haüy», en 


\section{VÍCTOR GUIJARRO MORA}

Esta desmedida confianza en la experiencia contrastaba con las actitudes de los matemáticos, quienes defendían más bien un uso restringido de estos métodos. Este era el caso de J. D'Alembert, para quien la claridad y la evidencia de una ciencia dependían del grado de generalidad y abstracción que tuvieran sus principios; a medida que añadiésemos propiedades sensibles, aumentaría proporcionalmente la confusión ${ }^{21}$. Por tanto, para uno de los editores de la Encyclopédie, el experimento tenía un escaso valor, sólo servía para contrastar la teoría y para cuestiones derivadas de ésta, por ejemplo, para problemas relacionados con los efectos del rozamiento o con la aerometría ${ }^{22}$.

No era este uso limitado de la experiencia el que inspiraba los compromisos de los físicos experimentales; para ellos los principios generales o básicos también debían estar basados en las impresiones sensoriales y su proximidad a ellas garantizaba su veracidad. En definitiva, se trataba de seguir fielmente las prácticas de dos autores del siglo XVII, el mencionado Boyle y J. Rohault. El filósofo natural inglés reconocía la importancia de la aproximación matemática, ejemplificada en los resultados de Galileo y Pascal, sin embargo no pensaba que ésta fuera el procedimiento adecuado para el conocimiento de la naturaleza ${ }^{23}$, o más bien para el descubrimiento de los agentes que son responsables de la producción de las cualidades que observamos. Para este cometido, además de los elementos enunciados, era preciso tener en cuenta entidades sensibles que fueran detectables mediante los aparatos. Esta era la característica que tenía el aire frente al éter, cuya existencia y efectos intentó comprobar ${ }^{24}$. En el caso del físico francés, Rohault, la experimentación adquirió un matiz diferente. Su punto de partida era estrictamente cartesiano, y de acuerdo con él, el mundo microfísico era inaccesible, no podemos conocer los componentes de la materia; por

GiLlisPIE, Ch. C. (1981), ed., Dictionary of Scientific Biography, Nueva York, Charles Scribner's Sons, vols 5-6, p. 178; para J. L. Heilbron, en la obra se hace un uso moderado aunque eficaz de las matemáticas, Heilbron, J. L. (1992), «Fisica e astronomia nel Settecento», Storia delle Scienze. Le scienze fisiche e astronomica, Torino, Giulio Einaudi editore, p. 331.

21 Encyclopédie de Diderot et d'Alembert, París, 1751, vol.13, «Discourse préliminaire des éditeurs», p. 7; Traité de dynamique, París, 1758, pp. I-II, y Essai sur les éléments de philosophie, Amsterdam, 1773 , vol. $4^{\circ}$, pp. 180-181.

22 Técnica iniciada en el siglo XVII para la determinación del peso específico de un cuerpo. En el XVIII, los principales modelos para llevar a cabo estas operaciones fueron ideados por D. G. Fahrenheit (descrito en FAHRENHEIT, R. H. (1724), «Araeometri novi descriptio \& usus», Philosophical Transactions, 33, 140-141) y W. Nicholson (descrito en NiCHOLSON, W. (1785), «New Instrument for Measuring the Specific Gravity for Bodies», Memoires of the Manchester Literary Philosophical Society, 2 , 386-396).

23 Más detalles sobre las posiciones metodológicas de Boyle, HALl, M. B. (1965), 55-56; Solís, C. (ed.) (1985), «La filosofía experimental», Robert Boyle: Física, química y filosofía mecánica, Madrid, Alianza, especialmente las pp. 21-27; y SARGENT, R.-M. (1995), The Dissident Naturalist. Robert Boyle and the Philosophy of Experiment, Chicago-Londres, The University of Chicago Press, 64-69.

24 SHAPIN, S y SCHAFFER, S. (1985), Leviathan and the Air-Pump: Hobbes; Boyle and the Experimental Life, Princeton, Princeton University Press, p. 181. 
tanto para ofrecer una explicación de los efectos observados, debemos obrar mediante hipótesis, que deben estar sujetas a su vez a principios metafísicos ${ }^{25}$. Una hipótesis sería pues un modelo mecánico en el que se emplean los objetos de la experiencia, ya que éstos no difieren de las partículas inaccesibles nada más que en cuanto a su tamaño. En conclusión, «confirmación» para un cartesiano no significaba lo mismo que para Galileo o Pascal, más bien se entendía como la posibilidad de encontrar un mecanismo a gran escala que por analogía representase los procesos microfísicos responsables de los efectos que observamos.

Por tanto, el descubrimiento de propiedades, de sus constancias y relaciones, la concepción de modelos explicativos, así como las demostraciones experimentales se convirtieron en el leit motiv de los físicos de la Ilustración. Para llevar a la práctica estas ideas generales los autores comprometidos con esta orientación se apoyaron en dos elementos fundamentales: las propiedades mecánicas anteriormente enunciadas y las analogías (basadas a su vez en el presupuesto metafísico de la uniformidad de la naturaleza). Así pues, por un lado, los procesos internos estaban condicionados por la base empírica que podíamos obtener en nuestras observaciones y que nos informaba aproximadamente sobre la posición y movimiento de las partículas. Por otra parte, el exigible nivel de generalidad de toda explicación física se conseguía gracias a que solamente se empleaba un puñado de propiedades (masa, posición, y algunas derivadas, como la elasticidad), de las que dependía la amplia variedad de las cualidades sensibles. El experimento, parte fundamental de esta postura epistemológica, servía para confirmar la verosimilitud del mecanismo propuesto.

Dentro de esta línea, las matemáticas cumplían un papel específico y limitado: reflejar mediante proporciones las regularidades observadas, y solamente éstas. Por tanto, las variables no representaban cuantitativamente alguna de las propiedades generales, como hacían los «físicos experimentales matemáticos», ya que esto supondría tomar como fundamental para la explicación algo inaccesible, ideal, hipotético y fuera del alcance de nuestros sentidos o de la capacidad de detección mediante los aparatos. Esta discrepancia respecto del alcance de las matemáticas es la que explica, por ejemplo, la diferencia entre los estudios de Musschenbroek, un físico experimental en sentido estricto, y los de Ch. Coulomb, un representante de lo que se he calificado como «física experimental matemática», en relación con la fuerza magnética. Para el primero, la fuerza se define a partir de sus manifestaciones externas,

\footnotetext{
25 Esto es, admitir, entre otros principios, que no hay nada en la naturaleza que no tenga propiedades, que todo efecto presupone una causa y que un cuerpo en movimiento no puede comunicar un efecto mayor que el que él mismo posee. Centrándonos en las propiedades, las fundamentales para Rohault son: solidez, dureza, impenetrabilidad y forma, teniendo en cuenta, primero, la identificación de la materia y la extensión (negación del vacío), y segundo, la indefinible división de la materia. Sobre el experimentalismo de corte cartesiano, Clarke, D. M. (1989), Occult Powers and Hypothesis. Cartesian Natural Philosophy under Luis XIV, Oxford, Oxford University Press.
} 


\section{VÍCTOR GUIJARRO MORA}

nada podemos conocer acerca de su naturaleza. Por tanto, en el caso del magnetismo, la vía para determinar sus efectos es una estrategia experimental consistente en un dispositivo compuesto por una balanza y dos piedras imán; la acción o la fuerza entre éstas últimas se contrarrestaría con la de la gravedad ${ }^{26}$ (véase la fig. 1). Así, para el físico holandés, a la hora de determinar la fuerza, debemos atender al efecto global producido por el objeto que tiene esta propiedad. En cambio, la propuesta de Coulomb partía de la consideración de cada uno de los polos como centros de fuerza separados, paso previo para lograr su finalidad: la determinación de la fuerza entre las partículas del fluido magnético (véase la fig. 2). Esto significaba atribuir una propiedad constante a una entidad microfísica, fuera del alcance de los sentidos, siendo las manifestaciones externas una consecuencia de esta peculiaridad de la materia ${ }^{27}$. De aquí que los experimentos estén orientados a aislar las unidades donde está concentrada la fuerza en el trozo de imán, que equivalen a la acción de una partícula, y medir sus efectos.

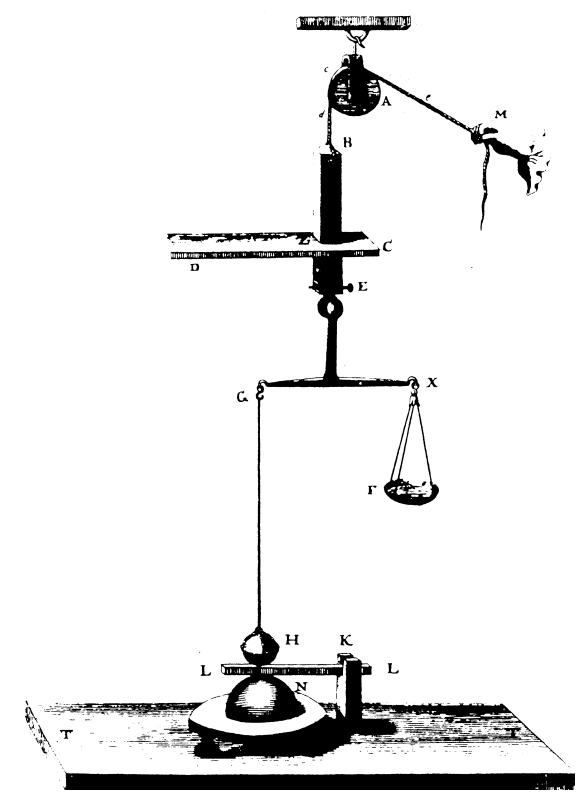

Fig. 1. Balanza magnética de Musschenbroek.

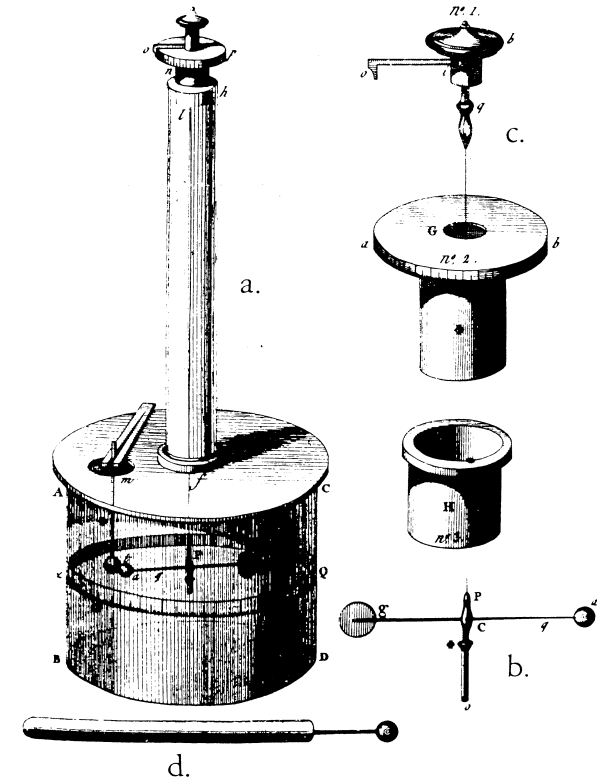

Fig. 2.

26 Procedimientos similares, sugeridos por J. Ellicot (1746), se pusieron en práctica para medir la fuerza eléctrica, WOLF, A. (1952, $2^{\mathrm{a}}$ ed.), A History of Science, Technology and Philosophy in the Eigtheenth Century, Londres, George Allen \& Unwin Ltd, p.254.

27 Estas diferencias de planteamiento ya fueron puestas de manifiesto por KUHN, T. S. (1961), «The Role of Measurement in the Development of Natural Science», Isis, 52, pp. 161-190; versión española en Kunn, T. S. (1983), La tensión esencial, México, Fondo de Cultura Económica, pp. 202-247. 
En el caso del estudio de la dilatación de los metales también se pusieron en práctica los dos procedimientos mencionados, esta vez teniendo como protagonistas al propio Musschenbroek y J. H. Lambert (un físico experimental matemático). Nuevamente el físico holandés pretende, sin conseguirlo, determinar una ley a partir de las observaciones obtenidas con un dilatómetro, donde las variaciones de longitud de las barras metálicas provocan el movimiento circular de una aguja sobre un cuadrante graduado (véase la fig.3). En este caso, el análisis del calor se reduce a la presencia de partículas dotadas de movimiento que producen un aumento de volumen en el cuerpo donde se alojan (mínima base física) y especialmente a las regularidades observadas con los aparatos; en ningún momento por tanto se aventura una propiedad matematizable inherente a estos corpúsculos. En cambio, Lambert sí intenta esta última aproximación tanto en sus análisis del equilibrio de temperaturas como en el de los cambios de volumen provocados por el calor ${ }^{28}$, donde parte de variables como el número de partículas de calor que existen en un cuerpo y pueden pasar a otro, la fuerza de cada una de ellas y el volumen de los cuerpos ${ }^{29}$.

Si bien los ejemplos vistos nos sirven para comprender algunos procedimientos de la física experimental, la finalidad primordial de ésta no fue el establecimiento de leyes, sino, como ya habíamos dicho antes, ofrecer dentro del marco conceptual corpuscularista un modelo mecánico que diera cuenta de los hechos. Estos modelos mecánicos estaban basados en la experiencia diaria y en la analogía, es decir, se extrapolaban datos observacionales a las descripciones de los procesos microfísicos. Los instrumentos, los aparatos y los dispositivos experimentales permitían, por un lado, manipular y controlar variables, y por el otro, dentro de esta tendencia, servían para reproducir procesos reales significativos. Para M. van Marum, director de la Fundación Teyler (Haarlem) ${ }^{29}$, la chispa producida por un generador probaba la existencia de un solo fluido;

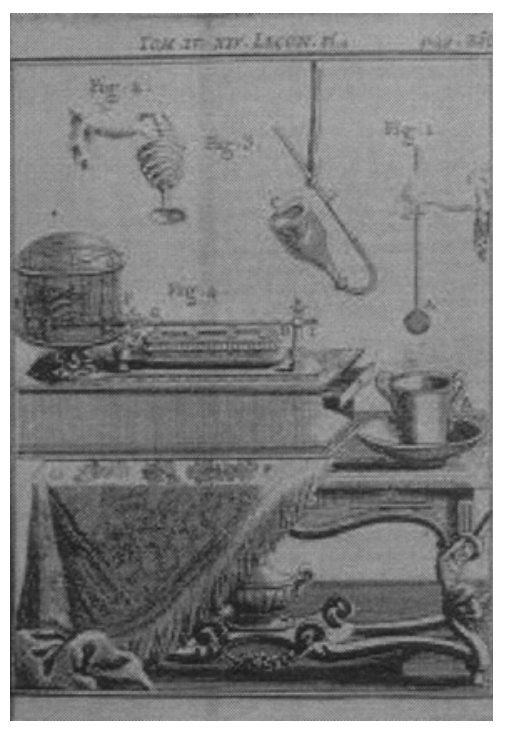

Fig. 3

\footnotetext{
28 Resultados que publicó en LAMBERT, J. H. (1755), «Tentamen de vi caloris, qua corpora dilatat, eiusque dimensione», Acta helvetica, physico-mathematica-anatomico-botanico-medica, 2, 172-242; sobre este tema, Fox, R. (1979), «The Science of Fire: J. H. Lambert and Study of Heat», Colloque International et Interdisciplinaire Jean-Henri Lambert, Mulhouse, 26-30 septembre (1977), París, Editions Orphyris, pp. 325-342.

29 Su interés por la adquisición de grandes máquinas eléctricas, que encargó en 1785 al mejor fabricante en esos momentos, el inglés J. Cuthberston, tenía la finalidad de producir grandes efectos para obser-
} 


\section{VÍCTOR GUIJARRO MORA}

para J. A. Nollet, entre la electricidad y el fuego existía necesariamente una analogía que se ponía de manifiesto en la capacidad de las chispas para inflamar el alcohol ${ }^{30}$. Por otra parte, la disposición de las limaduras de hierro sobre una lámina, sometidas a la acción de un imán, se convirtió en una imagen permanentemente presente en la mente de los físicos a la hora de dar cuenta de los mecanismos que justificaban los fenómenos magnéticos.

\section{CASOS Y RESULTADOS EN LA FÍSICA PARTICULAR Y EN LA GENERAL}

\section{Física particular (electricidad y calor)}

Dentro de los parámetros de la física experimental se produjo un importante impulso de los ámbitos incluidos, según la clasificación anterior, bajo la física particular. La electricidad, el estudio del aire, el calor, el magnetismo y la cohesión fueron las disciplinas en las que se pusieron en práctica las ideas que se han venido exponiendo.

La electricidad constituye el caso mejor estudiado, y dentro de este tema destacan, por la atención recibida, las figuras de B. Franklin y A. Volta. En el caso de Franklin, la finalidad primordial de su trabajo experimental entre 1747 y 1755 no fue la medición sistemática, sino la localización de la carga en la botella de Leiden así como la explicación del comportamiento de este dispositivo. También demostró que el relámpago era un fenómeno eléctrico, estudió la transmisión de la carga así como la descarga violenta. El resultado fue una extensamente empleada teoría de la acción eléctrica y numerosas reglas, que tenían, por ejemplo, la siguiente forma: «cuando un cuerpo contiene más electricidad en relación con su tamaño que otro, entonces, al unir ambos mediante un conductor o cuando se disponen suficientemente próximos para que salte entre ellos una chispa, el fluido se transmitirá uniformemente de uno a otro hasta que esté uniformemente distribuido entre los dos». Volta, por su parte, tampoco estaba interesado en la cuantificación ${ }^{31}$; sus esfuerzos (entre 1775 y 1805) se destinaron, en cambio, al análisis de la relación entre capacidad, tensión y carga, a la

observar más claramente los fenómenos asociados con la electricidad. Para Ch. Lichtenberg, otro de los partidarios de estas posturas, «Repetir un experimento con aparatos de mayor tamaño es equivalente a ver los fenómenos a través de un microscopio», cit. en BILANUIK, O. M., «G. Ch. Lichtenberg», GILLISPIE, CH. C., ed (1981), Dictionary of Scientific Biography, Nueva York, Scribner \& Son, vol. 8, p. 322.

30 Home, R. W. (1979), «Nollet and Boerhaave: A Note on Eigtheenth Century Ideas about Electricity and Fire», Annals of Science, 36, pp. 171-175, 173

31 Recibiría, sin embargo, la influencia, por un lado, de la Optica de Newton y los trabajos de Boscovich y Leibniz, y por el otro, de la orientación experimental-especulativa y la de la química de las afinidades, L. Fregonese, «Two different scientific programmes: Volta's electrology and Coulomb's electrostatics», en BlONDEL, Ch. y DÖRRIES, M. (1994), eds., Restaging Coulomb, Florencia: Leo S. Olschki, pp. 96-98. 
sustitución del concepto de atmósfera eléctrica por el de «esfera de actividad», y en conexión con la explicación del galvanismo, a la detección de cargas débiles mediante electroscopios (investigaciones cruciales para sus estudios sobre la electricidad producida en los contactos entre metales y, por tanto, para la invención de la pila). El resultado fue la definición de nuevos conceptos y sus relaciones, la invención del electróforo y el descubrimiento y estudio de la fuerza electromotriz (base de la pila).

Los trabajos de ambos autores proporcionan información sobre los procedimientos empleados en la explicación de los fenómenos naturales. En primer lugar, la utilización de los fluidos constituía la mejor manera de justificar las observaciones. Estos consistían, como ya se había dicho, en sustancias con propiedades físicas aunque no añadían masa; se movían, actuaban por contacto, se alojaban en los poros de los cuerpos, y en algunos casos manifestaban fuerzas de repulsión entre sus partículas. También podían transmitirse de un cuerpo a otro, se podían almacenar en ciertas circunstancias y la intensidad del efecto era proporcional a su cantidad o bien a su densidad. Con estos presupuestos se elaboraron los modelos basados en las analogías con el comportamiento hidrostático e hidrodinámico propio de la mecánica de fluidos. Por ejemplo, el generador eléctrico era comparado con una bomba aspirante que «extraía» la electricidad de la tierra y la transmitía hacia el conductor principal ${ }^{32}$. Para Franklin, la materia ordinaria era como una esponja para el fluido eléctrico; una vez que se saturaba, el fluido se distribuía alrededor del cuerpo formando una «atmósfera eléctrica» (similar por otra parte a la atmósfera terrestre). Este elemento fue empleado por el científico de Filadelfia para explicar las atracciones y repulsiones observadas en el nivel macroscópico, teniendo en cuenta que, en general, se descartaba por ininteligible la acción de fuerzas a distancia. Similares recursos a acciones por contacto, así como a imágenes basadas en la dinámica de fluidos, eran reclamados en los modelos concebidos por otro destacado investigador de la electricidad en el XVIII, J. A. Nollet. Para el francés, cuando se frotaba un cuerpo, salía de sus capas superiores un efluvio cuyo ingrediente fundamental era el fuego. Como resultado de esta expulsión súbita, quedaban en el cuerpo espacios que se llenarían con corrientes procedentes de los cuerpos circundantes. La acción de estos flujos y reflujos sobre los materiales ligeros explicaría los movimientos que observamos. En definitiva, la electricidad era el resultado de la alteración de la situación de equilibrio del fluido existente en un objeto.

En el caso de Volta, éste puso en práctica otro de los recursos mencionados anteriormente. Se trataba del uso de aparatos para ejemplificar y observar directamente fenómenos relevantes asociados con las consecuencias de una explicación teórica. Los útiles empleados para esta ocasión fueron el electróforo, cuya acción mostraba

32 HaCkMANN, W. (1978), Electricity from Glass. The History of the Frictional Electrical Machine (1600-1850), Alphen aan den Rijn, Sijthoff \& Noordhoff, p. 9. 


\section{VÍCTOR GUIJARRO MORA}

que la electricidad no desaparecía con la descarga (en contra de G. Beccaria), y el condensador, que hacía visibles los efectos de la electricidad, los cuales por su debilidad se escapaban del alcance de los sentidos ${ }^{33}$.

Los estudios asociados con el calor suministran igualmente muestras de los procedimientos empleados por los físicos experimentales. Sin embargo, entre este ámbito y la electricidad existió una diferencia importante: la disposición de un instrumento de medida fiable, como el termómetro, que dentro del segundo campo no existió. Solamente el empleo de alguno de los diversos tipos de electrómetro (realmente un electroscopio) hubiera representado un cambio sustancial dentro de las investigaciones eléctricas, pero, primero, carecían del requisito de linealidad, y segundo, tampoco estaba muy claro qué medían dentro del contexto de los modelos al uso ${ }^{34}$.

Entre estos físicos experimentales se asumió en general, y especialmente a partir de la publicación de los Elementa chemiae de H. Boheraave (1732), la consideración del calor como una sustancia, es decir, la idea de que éste era un tipo de fluido elástico presente en todos los cuerpos, compuesto de partículas atraídas por la materia ordinaria y que se repelían entre sí. De esta manera, los trabajos termométricos contaban con una mínima base física. Estos se habían iniciado tomando la variación del volumen de un cuerpo como fundamento de las constancias asociadas con una propiedad, una elección arbitraria ${ }^{35}$. A partir de ese momento, los esfuerzos se encaminaron hacia el análisis de las propiedades de la sustancia (aire, agua, alcohol, mercurio) empleada en las observaciones, y a la consecución de un instrumento fiable, factor no dependiente tanto de su sensibilidad como de la constancia y regularidad de su comportamiento, así como de la previsión de las imprecisiones. En torno a 1740 existían termómetros comparables, hecho que facilitaba el intercambio de observaciones y la confianza en las mediciones.

Dentro del ámbito del estudio de las constancias, y contando con el instrumento de medición, pueden observarse anomalías, irregularidades que se intentan incorporar a los rudimentos teóricos iniciales o que representan una ampliación de los mismos. Estas circunstancias fueron las que motivaron los trabajos de J. Black relacionados con el calor latente y con los problemas de cambio de estado, particularmente los de sólido a líquido. Para el autor inglés, siguiendo en esto a Boerhaave, los registros del termómetro eran el único indicador objetivo de la presencia de calor; sin embargo, y aquí se separaba del químico holandés, no constituían una medida de una

33 Sobre las estrategias de Volta y el uso de instrumentos, M. PERA, M. (1986), La rana ambigua, Torino, Giulio Einaudi (según versión inglesa de 1992, The Ambiguous Frog. The Galvani-Volta Controversy on Animal Electricity, Princeton, Princeton University Press, pp. 45-53).

34 Véase HackmanN, W., «Electroscope» y «Electrometer», en BUD, R. y WARNER, D. J., eds. (1998), Instruments of Science. An Historical Encyclopedia, Nueva York, Garland.

35 Middleton, W. E. K. (1966), A History of the Thermometer, Baltimore, The Johns Hopkins Press, pp. 48-49. 
cantidad sino de una intensidad ${ }^{36}$. Con estos puntos de partida realizó los experimentos sobre mezclas de agua congelada y líquida, teniendo en cuenta que agua=hielo + materia de calor y que vapor=agua+materia de calor. Así, observó que al dejar pesos iguales de hielo y agua en una habitación a una temperatura conocida, el primero tardaba 21 veces más el tiempo empleado por el segundo en alcanzar la misma temperatura del entorno; como el ascenso había sido de 33 a 40 grados F, entonces en total se habían necesitado $21 \mathrm{x}$ (40-33) «grados de calor», de los cuales 8 solamente se empleaban para el cambio térmico, y los restantes 137 para el cambio de estado. Por tanto, a través de estos planteamientos llevados a cabo en 1762, exentos de instrumentos de alta sensibilidad, y con el uso de simples operaciones aritméticas, se había detectado la presencia del calor latente, y además se habían conseguido cuantificar con cierta aproximación sus efectos, por ejemplo, en el caso de las mezclas de sustancias a diferente temperatura.

Así pues, la disposición de un instrumento fiable dentro de un ámbito determinado de la física constituía una herramienta poderosa para llevar a cabo análisis de las consecuencias vinculadas con un constructo teórico, así como para perfilar éste mediante el descubrimiento de propiedades que explicaran las anomalías. Además, el propio utillaje, en este caso el termómetro, entraba a formar parte del propio dispositivo experimental empleado para examinar los fenómenos, no concibiéndose tanto como un útil objetivo e independiente de la teoría cuanto como un mecanismo práctico que permitía observar directamente las evoluciones y funcionamiento de un modelo ${ }^{37}$. En conclusión, el aumento o disminución del volumen del mercurio en el tubo de vidrio no era el esperado a partir de una relación simple mecánica derivada de la cantidad del calórico, por tanto habría que pensar en la existencia de un factor adicional (el calor latente) para dar cuenta de e integrar las nuevas observaciones en la teoría.

En general, los modelos teóricos aportaban una mínima base física a las observaciones, no obstante mantenían una conexión débil con éstas, constituyendo más bien propuestas ad hoc versátiles y sujetas a la corrección en función de los descubrimientos. Este era uno de los puntos más frágiles de la física experimental, que se puso de manifiesto en todos sus campos. Por ejemplo, continuando con el último de los temas tratados, algunas sospechas teóricas junto con el examen de diversos fenómenos motivaron la revisión de las concepciones de la materia vinculadas con el fluido del calórico. Lo que estas concepciones no habían previsto eran ciertas anomalías relacionadas con la diferencia en el comportamiento del calor procedente de la llama y el

36 SCHOFIELD, opus cit., p. 187.

37 Se trataba por consiguiente de lo que W. Hackmann denomina «instrumento activo» (por oposición a los «instrumentos pasivos», como los relojes y las balanzas) que posibilitan la reproducción de las operaciones naturales, véase «Scientific Instruments: Models of Brass and Aids to Discovery», GooDING, D. et al., (eds.) (1989), The Uses of Experiment, Cambridge, Cambridge University Press, pp. 3165, y «The Relationship Between Concept and Instrument Design in Eighteenth-century Experimental Science», Annals of science, 36 (1979): 205-224. 


\section{VÍCTOR GUIJARRO MORA}

procedente del Sol; mientras el primero perdía gran parte de su intensidad al atravesar el vidrio, el segundo no, aunque, eso sí, la luz se transmitía sin apenas alteración en los dos casos. Además ¿cómo era posible pensar en un fluido que atravesara la enorme distancia existente entre la Tierra y el Sol? Los primeros experimentos que pusieron de manifiesto las disparidades anteriores fueron los realizados por E. Mariotte (1679), que posteriormente serían confirmados por R. Hooke $(1682)^{38}$, ensayos que constituyeron un precedente de los estudios asociados con el calor radiante. Hasta la década de los años 60, ya en el siglo XVIII, no se produjeron aportaciones significativas dentro de este ámbito. En concreto, para dar cuenta de las aceptadas diferencias y semejanzas entre la transmisión del calor y de la luz, así como de la incuestionable propiedad reflectiva del «calor oscuro», recurrentemente contrastada en el nivel experimental, se mantuvieron dos posturas con respecto a la naturaleza del calor radiante: que consistía en una emanación material (M.-A. Pictet), o bien que se trataba de la vibración de un fluido calórico presente en todos los cuerpos $(\mathrm{H}$. B. Saussure y B. Thompson ${ }^{39}$.

Por consiguiente, a finales de siglo se contaba dentro de los fenómenos conectados con la electricidad, el calor, el magnetismo, el aire y el agua con varias reglas prácticas, con el descubrimiento de nuevas propiedades y con un puñado de leyes y proporciones referidas, por ejemplo, al resorte del aire, el equilibrio térmico y la capacidad de los conductores. Además, en el terreno de los constructos teóricos, existían diversas propuestas para dotar de coherencia a los datos y experimentos: uno o dos fluidos en el caso de la electricidad y el magnetismo; en cuanto al calor, un fluido altamente elástico, compuesto de partículas autorrepulsivas, que se transmitía de un cuerpo a otro, o bien una materia presente en todos los cuerpos que actuaba en función de su vibración. Sugerencias similares, con algunas mínimas variaciones, encontramos también en el estudio del aire.

\section{FÍSICA GENERAL EXPERIMENTAL}

Según el esquema referido a la obra de Musschenbroek, la física experimental estaba dividida en una parte general y otra particular. Hasta ahora nos hemos referido a la segunda de las divisiones, donde se abordaron las problemas físicos inaprehensibles desde los planteamientos de las ciencias clásicas, dominadas por la mecánica, hidrostática e hidrodinámica. Sin embargo, en esta disciplina también se contemplaron, por un lado, los trabajos experimentales conducentes a demostrar en la práctica la validez de las concepciones teóricas basadas en la mecánica, y por el otro, las labores destinadas a aumentar la sensibilidad y precisión de los aparatos de medida

\footnotetext{
38 CORNELL, E. S. (1936), «Early Studies in Radiant Heat», Annals of Science, vol. I, p. 218

39 Ibid., 225.
} 
cuya finalidad no era la confección de teorías ni su cuestionamiento, sino también la resolución de determinados problemas prácticos. Precisamente este tipo de trabajos eran los característicos de la mecánica práctica, a la que se ha hecho referencia al principio del artículo, y por tanto diferenciable de lo que se ha venido describiendo como física experimental propiamente dicha. Así, en el primero de estos campos, el de la demostración de las concepciones teóricas, se enmarcan las minuciosas descripciones de W. J. 's Gravesande de la máquina de fuerza centrales, diseñada para comprobar las leyes fundamentales de la fuerza centrípeta, o bien los estudios de G. Atwood, quien también concibió una máquina («máquina de Atwood») para la confirmación de la segunda ley de Newton ${ }^{40}$. En ambos casos, y especialmente en el segundo, la precisión fue considerada un factor fundamental para la aceptación de las observaciones y los datos obtenidos ${ }^{41}$.

En el segundo de los ámbitos, el destinado a mejorar los aparatos, lo que interesaba, por ejemplo en el caso de dilatometría, era esencialmente confeccionar tablas que sirvieran para analizar las incorrecciones de los cronómetros debidas a la desigual dilatación de sus componentes de metal ${ }^{42}$. Eso sí, este interés impulsó la fabricación de dilatómetros provistos de una notable sensibilidad, como los elaborados por J. Ellicot (1736), J. Smeaton (1754), cuyos grados detectaban variaciones de 0,00012 cm., y J. Ramsden (c. 1780); en cambio ningún avance significativo se produjo a partir de estas labores en el conocimiento de los procesos internos del calor, los cuales, por otro lado, no hubieran requerido ese exageradamente alto índice de sensibilidad. Similares propósitos se adivinan en los esfuerzos por crear compases azimutales eficaces para medir las variaciones de la aguja en función de la observación de la posición del Sol a mediodía o de la estrella polar, observación que era comparada con la línea norte-sur de la brújula. Estas ocupaciones eran fundamentales para mediciones en tierra y para la realización de mapas de

40 Para Atwood (en ATwood, G. (1784), A Treatise on the Rectilnear Motion and Rotation of Bodies, Cambridge, p. 4) las leyes del movimiento se habían revelado ciertas tanto desde el punto de vista físico como del matemático. Para confirmar el primero de los requisitos el autor diseñó la máquina, cuya finalidad era comprobar que de acuerdo con la segunda ley dos masas, $\mathrm{m}_{1}$ y $\mathrm{m}_{2}$, se moverían según el siguiente valor de la aceleración: $\mathrm{a}=\mathrm{g}\left(\mathrm{m}_{2}-\mathrm{m}_{1} / \mathrm{m}_{2}+\mathrm{m}_{1}\right)$. Sobre los planteamientos de Atwood en este experimento, HANSON, N. R. (1977), Patrones de descubrimiento. Observación y explicación, Madrid, Alianza Universidad, pp. 205-208.

41 La máquina estaba ideada para anular los efectos del rozamiento en las poleas empleadas así como del peso de los hilos de los que colgaban las pesas y también de la acción del aire; por ejemplo, Sigaud de la Fond, en SIGAUd DE LA FOND, J.-A. (1777), Elémens de physique théorique et expérimentale, París, vol. I, descarta la importancia del peso del hilo afirmando que éste supondría en el recorrido total solamente una diferencia de 0,0312 segundos. Sobre la importancia de la precisión en este tipo de demostraciones, SHAFFER, S. (1994), «Machine Philosophy: Demonstration Devices in Georgian Mechanics», Osiris, 9, 157-182.

42 Sobre la evolución de la cronometría en el s. XVIII, DAUMAS, M., «La construction horlogère», en Daumas, M., ed. (1965), Historie Génerale des Techniques, París, P. U. F., Vol. 2, pp. 389-410, y sobre los diferentes modelos de dilatómetro, WOLF, opus cit., pp. 190-193. 


\section{VÍCTOR GUIJARRO MORA}

líneas isógonas, y sólo indirectamente se obtenía alguna ventaja para el conocimiento del magnetismo terrestre ${ }^{43}$. Igualmente, en el último cuarto del siglo XVIII, la realización de barómetros portátiles provistos de verniers para medir la altura de montañas impulsó la fabricación de instrumentos que eran capaces de ofrecer lecturas de 0,01 $\mathrm{mm}^{44}$, pero escasos conocimientos teóricos se derivaron de estas dedicaciones.

\section{CONCLUSIÓN}

Con esta última referencia se delimita aún más el alcance de la física experimental. En definitiva, ésta representó durante el siglo XVIII una opción dentro de las ciencias físicas cuyo pilar fundamental fue el fenomenalismo como vía de indagación de los procesos microfísicos y como principio demostrativo de la viabilidad de los presupuestos teóricos aplicados en los estudios naturales (por ejemplo, la naturaleza de la fuerza se muestra en sus efectos visibles). Como se ha visto, dentro de esta opción se impulsaron especialmente los estudios de física particular, donde se desarrollaron los trabajos asociados con fenómenos cuya explicación no se ajustaba a modelos basados exclusivamente en las leyes mecánicas generales.

Por estas razones, las matemáticas, que parten de principios universales, no tuvieron cabida en esta opción. Así pues, en general, se puede afirmar que sus planteamientos no se inspiraron en el legado newtoniano, ni en el de los Principios matemáticos ni en el de la Óptica ${ }^{45}$, sino que más bien se remiten a la herencia de personajes como Boyle, Hooke, Mariotte, Ricciolli, Grimaldi y Rohault. Se diferencian, por tanto, de las prácticas de los físicos experimentales matemáticos, como Lambert, Aepinus ${ }^{46}$, Cavendish ${ }^{47}$ y Coulomb, quienes sí podrían reclamar con justicia el califi-

\footnotetext{
43 Sobre esta cuestión, WARNER, D. (1994) «Terrestrial Magnetism: For the Glory of God and the Benefit of Mankind», Osiris, 9, pp. 67-84.

44 Sobre el desarrollo de estas técnicas, Feldman, T. S. (1985), «Applied mathematics and the quantification of experimental physics: the example of barometric hypsometry», Historical Studies in the Physical Sciences, 15, pp. 127-197.

45 En contra, en este último caso, de la opinión de I. B. Cohen expuesta en COHEN, I. B. (1956), Franklin and Newton. An Inquiry into Speculative Newtonian Experimental Science and Franklin's Work in Electricity as an Example thereof, Philadelphia, The Philosophical Society, p. 179.

46 Sobre la diferencia entre la aproximación del matemático alemán y la de otros autores del s. XVIII (como, por ejemplo, Musschenbroek) a los problemas eléctricos y magnéticos, HOME, R. W. «Physical Principles and the Possibility of a Mathematical Science of Electricity and Magnetism», en MÉtivier, M.; Pierre Costabel, P. y Dugac, P. (eds.) (1981), Siméon-Denis Poisson et la science de son temps, eds., París, École Polytechnique, pp. 151-166.

47 El científico inglés puso en práctica su inspiración newtoniana, basada en la fusión de la mecánica de fuerzas y la filosofía corpuscular, en los estudios sobre el aire, el calor (que concebía como el momento mecánico del movimiento vibratorio de las partículas) y en la electricidad, donde demostró que la fuerza eléctrica obedecía la ley del inverso del cuadrado; en relación con los presupuestos newtonianos
} 
cativo de herederos del filósofo natural inglés. En estos personajes se encuentran rasgos distintivos de la metodología e ideales que Newton puso en práctica en la Óptica, donde la finalidad no era tanto la combinación de especulación y experimento como la de buscar propiedades que admitiesen la matematización. Efectivamente, para el autor inglés, los análisis llevados a cabo en esta obra estaban destinados, una vez asumidas las conclusiones de la óptica geométrica, a estudiar las propiedades físicas de la luz, presuponiendo además un modelo de concepción de la materia basado en la hipótesis del corpuscularismo, es decir, en la idea de que los últimos componentes de la naturaleza son pequeños corpúsculos indivisibles dotados de masa y movimiento dentro del espacio vacío isótropo. El «experimento crucial», referente a la descomposición de la luz, se encuentra descrito en el libro primero (proposición II, exp.3), parte en la que previamente se han establecido definiciones y axiomas relativos a la reflexión y refracción de la luz, junto con sus ángulos, relaciones y proporciones, contenido que a su vez estaba matemáticamente asentado en las deducciones de la sección XIV del primer libro de los Principia. El experimento no aparece como un ensayo aleatorio, sino vinculado a presupuestos matemáticos y mecánicos que permiten, en este caso, conectar la producción de los colores con los diferentes índices de refracción de los distintos tipos de corpúsculos que componen la luz ${ }^{48}$, mostrándose experimentalmente que esta conexión es constante. De esta forma se introduce la matemática en las interpretaciones físicas. Cuando Newton plantea su famoso experimento de la descomposición de la luz lo que está intentando probar es que ésta no es una entidad simple, sino una mezcla de todos los rayos de colores, producidos por corpúsculos con alguna propiedad general. El prisma se limita a hacer visible el efecto de dispersión de los corpúsculos de cada tipo que contiene la luz. Complementando esta comprobación con los efectos derivados de la recomposición de los colores se podrán confirmar las hipótesis iniciales concebidas por el autor. Por tanto, a través del estudio de la luz, donde cuenta con la ventaja de conocer de antemano las leyes de la reflexión y la refracción, Newton pretende descubrir el comportamiento de los componentes de la materia, una intención que se hace también patente cuando por medio de los experimentos de interferencia (Libro II, Parte I) se decide a calcular el tamaño de los corpúsculos, o inferir de la velocidad de éstos los colores de los rayos.

Estos procedimientos coinciden con los que posteriormente ensayaron los físicos experimentales matemáticos, y que en algunos casos, como los de Aepinus, Cavendish y Coulomb, proporcionaron importantes resultados a la física. Sin embargo, no eran éstas las ideas que los físicos experimentales en sentido estricto tenían en la mente a la hora de emprender sus labores características. Para ellos el experimento no estaba destinado a distinguir alguna propiedad general, como hace Coulomb en el

de Cavendish, MCCORMmaCH, R. (1960), «Henry Cavendish: A Study of Rational Empiricism in Eighteenth-Century Natural Philsophy», Isis, 60, pp. 293-306.

48 Newton (1977), Optica, ed. de C. Solís, Madrid, Alfaguara, pp. 214-215. 


\section{VÍCTOR GUIJARRO MORA}

caso de la electricidad o el magnetismo, y que la balanza de torsión de hilos (como el prisma, en el caso de Newton) hace visible. Sus propósitos tenían otras finalidades, que pueden sintentizarse en: comprobar los constructos teóricos provisionales (hipótesis), detectar el efecto de propiedades, visualizar los fenómenos inaccesibles (como en el siglo XVII se había empleado el microscopio), y descubrir rasgos y peculiaridades de los fenómenos, irreductibles a la masa, velocidad o fuerza mecánica, que explicasen éstos.

En definitiva, la física experimental constituyó una manera específica de abordar los problemas físicos (y no la antesala de la física experimental matemática), aplicada preferentemente a las áreas mencionadas y con los métodos señalados, cuyo estilo se inicia en los tratados de Desaguliers y Hauksbee, se sistematiza en los manuales de Musschenbroek y culmina con Volta. Sus procedimientos condujeron a importantes descubrimientos y significaron un impulso extraordinario del método experimental, materializado en el desarrollo y mejora del utillaje de laboratorio y en la atención a las condiciones prácticas de aplicabilidad de los presupuestos teóricos. Pero el desmesurado énfasis en la información sensorial ${ }^{49}$ tanto para la confección de analogías y modelos explicativos como para la aceptación de hipótesis alternativas ${ }^{50}$, limitaron la consistencia, el alcance y la solidez de las teorías sugeridas en este ámbito. Precisamente la tendencia en el siglo XIX dentro de los estudios experimentales, ya iniciada a finales del anterior, fue la admisión de la separación entre modelos conceptuales y el mundo real, lo que dio lugar a teorías más flexibles cuyo punto de partida no debía necesariamente estar condicionado por la experiencia, sino únicamente sus consecuencias $^{51}$.

49 Que en muchas ocasiones estimuló el uso del experimento como espectáculo (véase RIDER, R. E., «El experimento como espectáculo», ORDOÑEZ, J. y ELENA, A., comps. (1990), La ciencia y su público, Madrid, CSIC, pp. 113-146) o su uso retórico (véase en este caso, SuTTON, G. V. (1995), Science for a Polite Society. Gender, Culture and the Demonstration of Enlightenment, Boulder (Colorado), Westview Press, pp. 191-240).

50 Hecho que conduciría a la incomprensión de otras propuestas, como la de Aepinus, por su exceso de abstracción y matematización; véase HOME, R. W. «Electricity in the Essay», en AEPINUS, T. A. (1979), Essay on the Theory of Electricity and Magnetism, introd. y notas de Home, Princeton, Princeton University Press, p. 113.

51 Por otra parte, la hostilidad hacia las matemáticas se mantendría en ese siglo entre los físicos alemanes; sobre este tema, CANEVA, K. L. (1978), «From Galvanism to Electrodynamics: The Transformation of German Physics and Its Social Context», Historical Studies in the Physcal Sciences, 9, 63-159. 